\title{
Clinical audit: long-term follow-up of women with genital lichen sclerosus
}

\author{
WC Ngu ${ }^{1 *}, \mathrm{C} \mathrm{Green}^{2}$ \\ From International Conference for Healthcare and Medical Students 2011 \\ Dublin, Ireland. 4-5 November 2011
}

\section{Introduction}

Genital lichen sclerosus (LS) is a chronic inflammatory disease usually managed with intermittent potent topical corticosteroids.5\% of women with untreated vulval LS go on to develop squamous cell carcinoma (SCC).

Current guidelines from the British Association of Dermatologists (BAD) state that patients on topical corticosteroids should be reviewed at least annually by their General Practitioner (GP) [1]. Previous work has shown that many women discharged from secondary to primary care are lost to follow-up [2].

\section{Methoda}

An anonymised questionnaire was sent to sixty women with genital LS discharged from our regional vulval clinic more than a year previously. Caldicott Guardian approval was obtained for the study.

\section{Results}

Forty-five patients (75\%) returned the questionnaire. Seventy one percent of patients had not attended their GP for follow-up. Of the 17 patients who had seen their GP, only 7 had had their vulval area examined. Only $53 \%$ of patients self-examined their vulval area. $48 \%$ were not aware of the need to report any area of persistent abnormality to their GP. 28 patients were still using topical corticosteroid - 24 of these potent or ultrapotent steroid. $48.9 \%$ of all respondents thought a $30 \mathrm{~g}$ tube of strong steroid should last a year, $26.7 \%$ three months, $13.3 \%$ one month and $11.1 \%$ were unsure. Only 30 patients $(66.7 \%)$ were aware of the increased risk of skin cancer associated with vulval LS, the remainder being unaware or uncertain.

\section{Conclusions}

Our study has highlighted worrying deficiencies in the long-term follow-up of this patient group. We have devised a brief patient information leaflet to be provided to both the patient and their GP at discharge to detail the small increased cancer risk, the need for monthly self-examination, clear instructions on the use of topical corticosteroids and the need for annual review by their GP. Hopefully, this will lead to an increased quality of patient care. We then plan to repeat the audit after an appropriate time interval.

\section{Author details}

${ }^{1}$ Ninewells Hospital \& Medical School, University of Dundee, UK. ${ }^{2}$ Ninewells Hospital \& Medical School, UK.

Published: 9 July 2012

References

1. Neill SM, Lewis FM, Tatnall FM, NH Cox: British Association of Dermatologists' Guidelines for the Management of Lichen Sclerosus 2010. BJD 2010, 147(4):640-649.

2. Rossi E, Pavanello P, Franchella A: Lichen sclerosus in children with phimosis. Minerva Pediatr 2007, 59(6):761-5.

doi:10.1186/1753-6561-6-S4-P45

Cite this article as: Ngu and Green: Clinical audit: long-term follow-up of women with genital lichen sclerosus. BMC Proceedings 2012 6(Suppl 4): P45.

${ }^{1}$ Ninewells Hospital \& Medical School, University of Dundee, UK

Full list of author information is available at the end of the article 